\title{
Research on the Reform of University Computer Courses with Cultivating "Computational Thinking" under the Background of New Engineering
}

\author{
Wang Xiaojie ${ }^{1, a}$,Ge Yanling 1,b,Wang Jiuru ${ }^{1, c}$, Ding Linhua ${ }^{1, d}$ \\ ${ }^{1}$ School of Information Science and Engineering, Linyi University, Linyi, Shandong, China \\ awangxiaojie@lyu.edu.cn, bgeyanling@lyu.edu.cn, `wangjiuru@lyu.edu.cn, dDinglinhua@lyu.edu.cn \\ * Wang Xiaojie
}

Keywords: new engineering, computational thinking, mixed teaching mode, flipping class.

\begin{abstract}
Under the background of new engineering, the demand for cross-integrated innovative talents has been increasing. The university computer courses have established a curriculum reform orientation with cultivating of "computational thinking". At the same time, a reform plan has developed with building a "wide-fledged" curriculum system, establishing "three field" teaching content, adopting the "mixed teaching mode" based on MOOC/SPOC+, flip classroom, and information teaching tools, and using the open and transparent "process evaluation system".
\end{abstract}

\section{新工科背景下以培养“计算思维”为核心的大学计算机课程改革研究}

王晓洁 $1, \mathrm{a}$ ，葛艳玲 ${ }^{1, b}$ ，王九如 ${ }^{1, c}$ ，丁林花 ${ }^{1, c}$

1临沂大学信息科学与工程学院, 临沂, 山东, 中国

awangxiaojie@lyu.edu.cn, bgeyanling@lyu.edu.cn, cwangjiuru@lyu.edu.cn, dDinglinhua@lyu.edu.cn *王晓洁

关键词：新工科；计算思维；混合教学模式；翻转课堂

中文摘要. 新工科背景下, 对交叉融合的创新型人才的需求不断增加, 大学计算机课程组确 立了培养“计算思维”为核心的课程改革定位，制定以构建“宽专融”课程体系，建立“三个领域” 教学内容体系, 采用MOOC+SPOC+翻转课堂+信息化教学工具的“混合教学模式”, 使用公开 透明的学生成绩“过程评价体系”为目标的改革方案。

1. 引言

新工科背景下，对交叉融合的创新型人才的需求日益增多。新的课程体系中计算思维能 力的培养和交叉课程的开设为培养学科融合的人才奠定了基础。

1.1 新工科背景下对跨专业, 学科融合型人才的需求是课程改革的契机。

相对于传统人才，“新工科”人才不仅在某一学科专业上学业精深，而且还应具有"学科交 叉融合"的特征; 不仅能运用所掌握的知识去解决现有的问题，也有能力学习新知识、新技术 去解决未来发展出现的问题。 


\section{2 教指委文件的发布确立了大学计算机基础教学课程体系改革的目标。}

2016年教育部高等学校教学指导委员会（下简称教指委）编制了《大学计算机基础课程 教学基本要求》（下简称《要求》），对大学计算机基础课程的提出构建“宽专融”课程体系 的要求。

\section{3 计算思维的提出标志着大学计算机基础教学内容改革进入新阶段。}

为了适应当前人才培养的需要, 迎接经济发展对人才培养的挑战, 课程组提出新工科背 景下以培养“计算思维”为核心的大学计算机“宽专融”课程体系改革研究。

\section{2. 改革方案设计}

确立了培养“计算思维”为核心的课程改革定位后, 课程组制定以构建“宽专融”课程体系, 建立“三个领域”教学内容体系, 采用MOOC+SPOC+翻转课堂+信息化教学工具的“混合教学模 式”，使用公开透明的学生成绩“过程评价体系”为目标的改革方案。如图1所示。

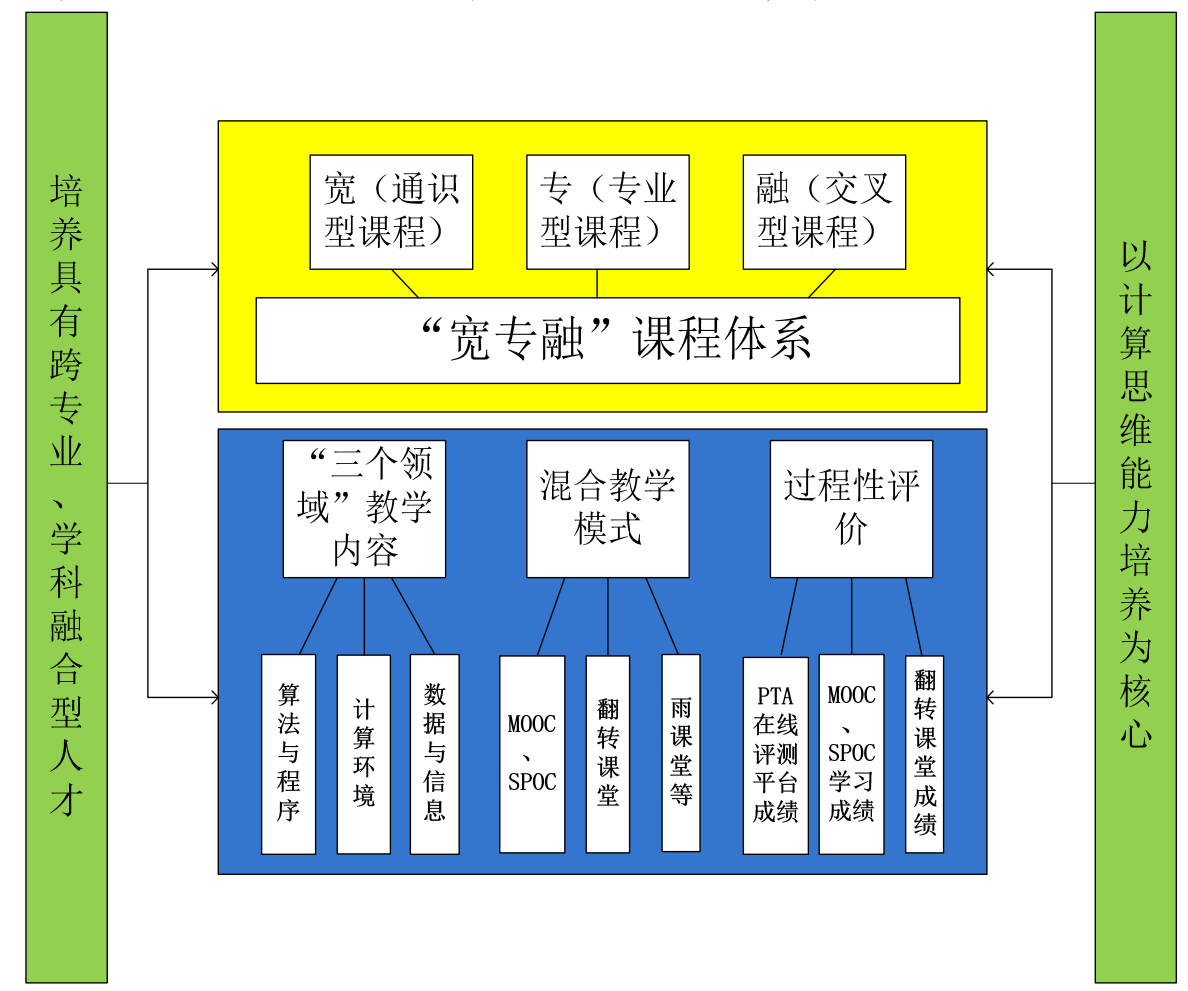

图1 实施方案

\section{1 构建“宽专融”兼顾的课程体系，满足专业交叉融合的需要。}

课程总体上可以分为“宽、专、融”三个类型：“宽”是指通识型（基础类）课程、“专”是 指专业型课程, “融”是指交叉型课程。

\section{2 建立“三个领域”课程内容体系, 将计算思维核心思想融入课程教学。}

《要求》中通过分类的“核心概念”来表达计算机思维的基本内涵，将大学计算机基础教 学的知识领域分为系统平台与计算环境、算法基础与程序开发、数据管理与信息处理三个领 域。

教师需要更深层次的理解计算机学科最本质的思想和方法, 以便在大量的知识体系中选 择出能够适合临沂大学学生的核心概念, 并在课程内容涉及和教学过程有重点的渗透这些思 想和方法。 
2.3 采用适合不同专业学生的混合教学模式, 提升人才培养质量, 培养新工科背景下适合区 域经济产业发展、具有学科交叉融合知识体系人才。

针对不同专业以及不同课程选取适合的教学模式, 注重学生的主体性、创造性、发展性、 整体性和差异性, 引导学生自己去学习探究, 培养学生自主探索、主动学习和合作学习的精 神。

2.4 改革学生成绩评价体系, 促进和激励学生能力提升, 及时改进课程教学效果, 为教学工 作的调整和改革提供依据。

在过程性评价体系中，根据不同课程从线上线下各成绩中选取真实性有效性程度高的、 系统即时评测的或者能即时发布随时查看的、能体现学生知识掌握能力到知识运用能力再到 创新能力培养的成绩纳入评价体系, 既全面公正的反映教学效果, 又保证成绩的公开透明。

\section{3. 解决问题的方法}

3.1 对区域产业经济发展对行业人才的具体需求、本校各专业学生对计算机类课程学习志趣、 专业教师对计算机类课程的要求进行调研和分析。

设计网上问卷与实地走访调查的方法对区域内企事业单位、各专业学生和教师对开设课 程、岗位需求、人才培养等进行调研。以供课程改革参考。

当前课程设置不能完全适合与学生志趣与区域经济发展对人才的需求, 还需要对比例和 内容进行进一步修改。

3.2 根据调研和分析结果和专业特点和人才培养方向, 构建新的课程体系、确立新的教学内 容体系。

3.2.1 构建兼顾“宽专融”的课程体系。

根据调研结果与各专业教师进行研讨，共同构建兼顾“宽专融”的临沂大学计算机基础课 程体系。尝试解决与专业融合度不够的问题。

自2017年开始采用第一学期分文、理、艺体三大学科开设全校必修的通识型“计算思维” 课程, 第二学期分专业开设专业型选修课, 交叉性课程根据课程特点灵活开设的课程体系, 兼顾“宽专融”，尝试解决与专业融合度不够的问题。

3.2 .2 确立以 “三个领域”为基础的内容体系。

根据临沂大学的人才培养目标和学生实际情况、围绕“计算机思维”核心，根据调研结果 选取相应必修与选修知识点, 对教学内容进行全面更新和重组, 并将移动互联网、云计算、 大数据等新概念添加到课程内容中，在课程中更好的渗透和强化这些“核心概念”。

3.3 针对不同专业特点和不同课程需求, 灵活运用MOOC+SPOC+翻转课堂+信息化教学工 具, 建立 $1+\mathbf{M}+\mathbf{N}$ 的混合教学模式。

3.3.1 选取适合的MOOC资源、以自建SPOC资源为补充，进行线上学习。

中国大学 MOOC、学堂在线、网易云课堂、北大的华为慕课等网站都有大量 MOOC 资 源, 自建《计算思维》《多媒体技术》《Python程序设计语言》《C 语言程序设计语言》等SPOC 资源。

3.3 .2 设计多种翻转模式充实翻转课堂, 进行线下学习。

翻转课堂向来是 MOOC 课程的有力支撑。本课题采用三种翻转形式: 课前随堂测验/考 试 (小翻转) 、课中/讨论 (中翻转) 、大作业 (大翻转)。 
3.3.3 使用雨课堂等信息化教学工具配合 SPOC与翻转课堂, 开展混合式教学。

雨课堂最大的便捷之处就是可以课下通过微信推送课件等资源和随堂测验, 尤其是随堂 测验既可以做成一个考试, 也可嵌入到PPT 中, 促进学生认真听课和思考问题, 测验结果一 目了然。

\section{4 根据不同教学模式制定合适的学生评价体系。}

不同的教学模式对应不同学生评价方式。平时成绩占学业成绩很大一部分比重, 一般不 低于 $40 \%$ 。

MOOC课程和SPOC课程根据线上学习情况和测试成绩直接计入评价体系。

翻转课堂成绩由教师评价、组间评价、组内评价比例三部分构成，现场评价并公布，保 证公平公开透明。

利用在线测试平台“PTA”进行期末考试（包含程序设计部分）考核。

在线评测平台不仅仅可以在线评测传统的主观客观题，对程序题也可以进行在线评测。 期末考试采用随机组卷, 并启动监考系统来最大程度保障学生成绩的真实性 。

\section{4.结语}

为了适应地方型高校人才培养的需要, 以培养“计算思维”为核心的大学计算机“宽专融” 课程体系改革研究将“计算思维”的培养完全融入大学计算机基础教学中。大学计算机课程组 明确了课程定位, 制定了新的课程体系, 采用新的教学模式, 使用新的过程评价体系。本文 从改革方案设计, 到具体采用的方法都进行了详细介绍, 对进一步培养学生“计算思维”能力 打下良好的基础。

\section{致谢}

本文为山东省高等教育本科教改项目 (M2018X058) 及临沂大学 2016 年度校级精品课程 《离散数学》、2017 年度校级教学质量工程项目在线开放课程《大学计算思维》、临沂大学 2018 年度校级教学改革与研究项目《新工科背景下以计算思维为核心的大学计算机“宽专融” 课程体系改革研究》的阶段性成果之一。

\section{References}

[1] Wang Wenjun, Wang Jing, Zhang Yee. The Positioning of Computer Basic Courses and the Construction of Curriculum System Based on Computational Thinking, Computer Education, vol.4, pp. 139-144, 2017.

[2] Wang Zhiqiang, Liu Fangfang. Research on Computer Basic Curriculum Reform Based on Computational Thinking, Decision Sciences, vol.17, pp. 292-311, 2013.

[3] Wang Juan, Lu Hua, Feng Yan.Thoughts on Computer Basic Education in Foreign Universities, Fujian Computer, vol.191, pp. 152-159, 2017.

[4] Zhang Dongsheng, Li Jie, Qiao Baojun. Graded Classification Teaching of Computer Basic Courses Based on Cultivating Computational Thinking, Computer Education, vol.9, pp. 10-12, 2015.

[5] Liang Hui, Exploration on Classification Teaching of Computer Basic Courses Based on Computational Thinking, Course Education Research, vol.7, pp. 243, 2013. 